Sir,

\section{New ocular phenotype associated with a mutation in the PAX2 gene}

Renal-coloboma syndrome (RCS) is an autosomal dominant disorder caused by PAX2 gene mutations and defined by the association of optic nerve coloboma and renal hypoplasia. ${ }^{1-3}$ We report a previously unknown phenotypic presentation of RCS in which calcified keratopathy and posterior lens luxation were associated with optic nerve defect.

\section{Case description}

A 10-year-old girl was referred to our hospital because of progressive loss of visual acuity in the right eye. Several years before, the child had been diagnosed as having RCS on the basis of renal hypoplasia associated with optic nerve colobomas. Genetic studies had confirmed the presence of a heterozygous PAX2 gene mutation: 619insG. The child had additional anomalies such as mental retardation, epilepsy, vesicoureteral reflux, and joint laxity. There was no history of ocular trauma, and no family history of renal or ocular disorder was identified. Examination under anaesthesia revealed severe calcified keratopathy of the right cornea (Figure 1). The lens did not seem to be present through the pupil of the right eye and fundus was hardly accessible because of corneal opacity, so that a B-scan ultrasonography was performed and showed posterior lens luxation (Figure 1). As expected, the left eye fundus revealed an optic disc coloboma.
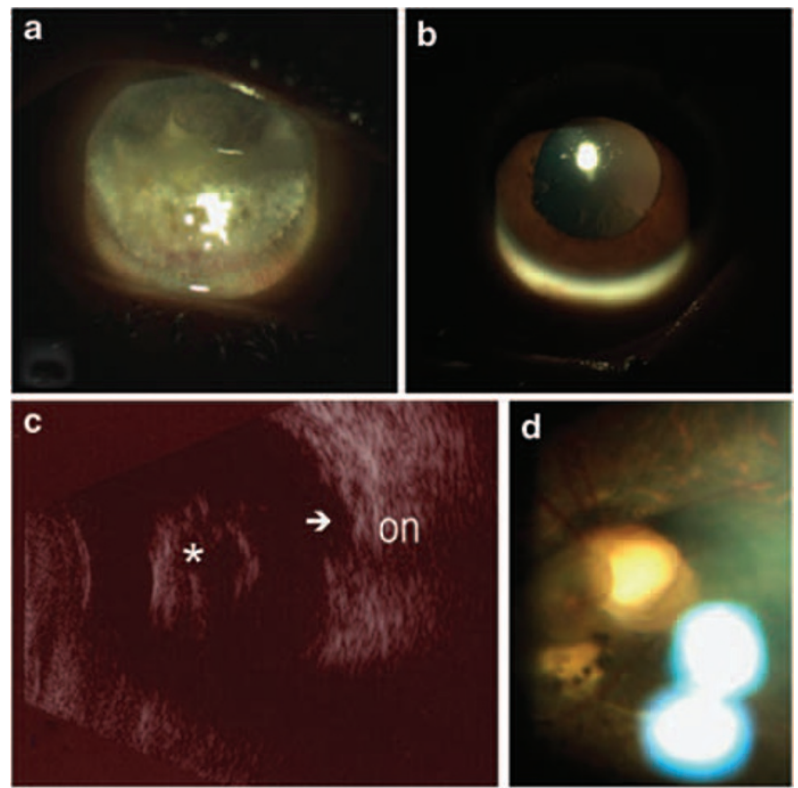

Figure 1 Unusual ocular phenotype associated with RCS and PAX2 mutation (a-d). Operating room photographs of right eye (a) and left eye (b). The right cornea presented with severe calcified keratopathy (a) whereas the left anterior segment appeared normal (b). (c) B-scan ultrasonography of the right eye showing lens dislocation (asterisk) in the vitreous and optic nerve colobomas (white arrow). on: optic nerve. (d) Photograph of left eye fundus showing optic disc coloboma.

\section{Comment}

In this case report, the extra-ocular phenotype is characteristic of RCS. Conversely, the phenotype exhibited on the right eye has not been previously reported. Calcified keratopathy, which represents the first corneal defect ever documented in RCS, is probably consecutive to chronic renal failure with calcium phosphate disturbances. ${ }^{4}$ The second intriguing ocular abnormality was the presence of spontaneous lens luxation. Interestingly, investigators have previously reported that patients with RCS may have joint abnormalities such as joint laxity. ${ }^{2,5}$ In this case, the child also suffered from joint laxity and had been operated on for bilateral hip dislocation. Therefore, we suggest the possibility that lens luxation could be part of a general disorder involving the connective tissue or elastic fibres of different types and locations. This original description stresses the importance of investigating potential cornea or lens damage in patients presenting with such a syndrome.

\section{Conflict of interest}

The authors declare no conflict of interest.

\section{References}

1 Schimmenti LA, Pierpont ME, Carpentier BLM, Kashtan CE, Johnson MR, Dobyns WB. Autosomal dominant optic nerve colobomas, vesicoureteral reflux, and renal anomalies. Am J Med Genet 1995; 59: 204-208.

2 Eccles MR, Schimmenti LA. Renal-coloboma syndrome: a multi-system developmental disorder caused by PAX2 mutations. Clin Genet 1999; 56: 1-9.

3 Parsa CF, Silva ED, Sundin OH, Goldberg MF, De Jong MR, Sunness JS et al. Redefining papillorenal syndrome: an underdiagnosed cause of ocular and renal morbidity. Ophthalmology 2001; 108: 738-749.

4 Aktas Z, Ozdek S, Asli Dinc U, Akyurek N, Atalay V, Guz G et al. Alterations in ocular strafcel and corneal thickness in relations to metabolic control in patients with chronic renal failure. Nephrology 2007; 12: 380-385.

5 Schimmenti LA, Cunliffe HE, McNoe LA, Ward TA, French $\mathrm{MC}$, Shim $\mathrm{HH}$ et al. Further delineation of renal-coloboma syndrome in patients with remarkable variability of phenotype and identical PAX2 mutations. Am J Hum Genet 1997; 60: 869-878.

F Beby ${ }^{1}$, O Roche ${ }^{2}$, P Cochat ${ }^{3}$, B Ranchin ${ }^{3}$, R Kohler $^{4}$, C Bonifas ${ }^{1}$, M-P Cordier ${ }^{5}$, T Attie-Bitach ${ }^{6}$, C Burillon ${ }^{7}$ and $P$ Denis ${ }^{1}$

${ }^{1}$ Department of Pediatric Ophthalmology, Femme Mère-Enfant Hospital, University of Lyon 1, Lyon, Rhône, France

${ }^{2}$ Department of Ophthalmology, Necker-Enfants Malades Hospital, Paris Cedex 15, France ${ }^{3}$ Department of Pediatric Nephrology, Femme Mère-Enfant Hospital, University of Lyon 1, Lyon, France ${ }^{4}$ Department of Pediatric Orthopedic Surgery, Femme Mère-Enfant Hospital, University of Lyon 1, Lyon, France

${ }^{5}$ Department of Genetics, Femme Mère-Enfant Hospital, University of Lyon 1, Bron Cedex, France ${ }^{6}$ Department of Genetics and INSERM U-781, Necker-Enfants Malades Hospital, Paris Cedex 15, France 
${ }^{7}$ Department of Ophthalmology, Edouard Herriot Hospital, Lyon Cedex 03, France

E-mail: beby.francis@neuf.fr

Eye (2010) 24, 1293-1294; doi:10.1038/eye.2009.330; published online 15 January 2010

\section{Sir, Conjunctival lymphoma following treatment with cyclophosphamide}

Conjunctival lymphomas typically result from a monoclonal B-cell proliferation and represent either localized disease or a systemic malignancy. ${ }^{1}$ Most patients are over 60 years of age or are immunosuppressed. ${ }^{1}$ Patients typically present with insidious onset of an asymptomatic, salmon-coloured, well-demarcated mass. Uncommonly, lymphoma may occur following treatment with immunosuppressive medications. ${ }^{2}$ We present a case of conjunctival lymphoma that presented 4 years after treatment with cyclophosphamide. Although the association may be coincidental, our case emphasizes that physicians should be alert to the possibility of secondary malignancy occurring in the eye following systemic immunosuppression.

\section{Case report}

A 68-year-old man, with a history of sympathetic ophthalmia that occurred 6 years earlier, noticed a painless red mass in his right eye. Therapy for sympathetic ophthalmia included prednisone and a 6-month course of cyclophosphamide $50 \mathrm{mg} /$ day. His past ocular history was otherwise notable for multiple intraocular surgeries in the right eye for cataract and open-angle glaucoma. On presentation, his visual acuity was $20 / 25$ OU. Examination of the right conjunctiva revealed a salmon-coloured, vascularized mass from 1 o'clock to 3 o'clock (Figure 1). Fundus examination revealed a sunset glow appearance and chorioretinal scars bilaterally.

Biopsy revealed a diffuse lymphocytic stromal infiltrate (Figure 1b). Most lymphocytes were enlarged, with variable cytoplasm, irregular nuclei, and intranuclear inclusions (Dutcher bodies).

Immunophenotyping revealed strong CD20 and kappa light chain positivity. The histopathological diagnosis was a low-grade B-cell lymphoma that was mostly consistent with marginal zone lymphoma. This type of lymphoma is often referred to as mucosa-associated lymphoid tissue lymphoma. Treatment involves evaluation for systemic disease. Localized conjunctival lymphoma may be treated with radiotherapy, although some cases may be cured by local excision.

\section{Comment}

Long-term studies of patients treated with cyclophosphamide showed an increased risk for malignancy, including lymphomas. ${ }^{24}$ Hoffman et $a l^{2}$ observed a 2.4-fold overall increase in the incidence of malignancies, and an 11-fold greater incidence of systemic lymphomas in the general population. The risk appears to be dose-dependent, particularly with cumulative doses $>50 \mathrm{~g}$, but is also reported with lower doses, and may

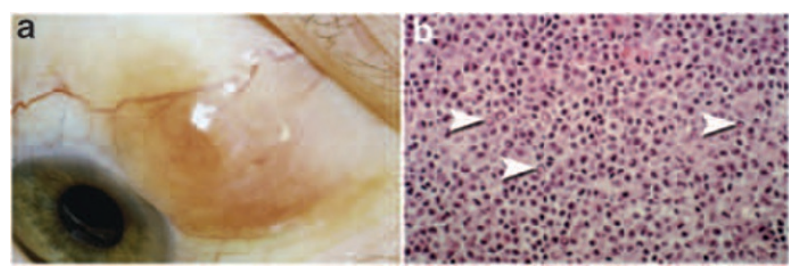

Figure 1 (a) Salmon-coloured, vascularized conjunctival mass of the right eye, extending from approximately 1 o'clock to 3 o'clock. Note the intact overlying conjunctiva and lack of feeder vessels. (b) Biopsy of the conjunctival mass, showing a nodular and diffuse lymphocytic infiltrate in the conjunctival stroma. Note the enlarged lymphocytes with variable cytoplasm and somewhat irregular nuclei. Higher magnification revealed intranuclear inclusions (Dutcher bodies, indicated by arrowheads). Most of the cells stained positively for CD20 and kappa light chains.

develop for up to 10 years. ${ }^{5}$ Our patient received a total of $9 \mathrm{~g}$. Potential mechanisms of malignancy include induced chromosomal alterations, altered immune-mediated anti-tumour surveillance, and increased susceptibility to oncogenic agents. ${ }^{3}$ Although the possibility remains that the association was spurious, our report of conjunctival lymphoma following treatment with systemic cyclophosphamide is the first report of such a secondary ocular malignancy, and illustrates that physicians should be alert to the possibility of secondary malignancy occurring in the eye following systemic immunosuppression.

\section{Conflict of interest}

The authors declare no conflict of interest.

\section{Acknowledgements}

This work was supported in part by The San Francisco Retina Foundation and The Pacific Vision Foundation.

\section{References}

1 Shields CL, Shields JA, Carvalho C, Rundle P, Smith AF. Conjunctival lymphoid tumors: clinical analysis of 117 cases and relationship to systemic lymphoma. Ophthalmology 2001; 108(5): 979-984.

2 Hoffman GS, Kerr GS, Leavitt RY, Hallahan CW, Lebovics RS, Travis WD et al. Wegener granulomatosis: an analysis of 158 patients. Ann Intern Med 1992; 116(6): 488-498.

3 Radis CD, Kahl LE, Baker GL, Wasko MC, Cash JM, Gallatin A et al. Effects of cyclophosphamide on the development of malignancy and on long-term survival of patients with rheumatoid arthritis. A 20-year followup study. Arthritis Rheum 1995; 38(8): 1120-1127.

4 Kempen JH, Gangaputra S, Daniel E, Levy-Clarke GA, Nussenblatt RB, Rosenbaum JT et al. Long-term risk of malignancy among patients treated with immunosuppressive agents for ocular inflammation: a critical assessment of the evidence. Am J Ophthalmol 2008; 146: 802-12.e1. Jun 23.

5 Travis LB, Curtis RE, Glimelius B, Holowaty EJ, Van Leeuwen FE, Lynch $\mathrm{CF}$ et al. Bladder and kidney cancer following cyclophosphamide therapy for non-Hodgkin's lymphoma. J Natl Cancer Inst 1995; 87(7): 524-530. 